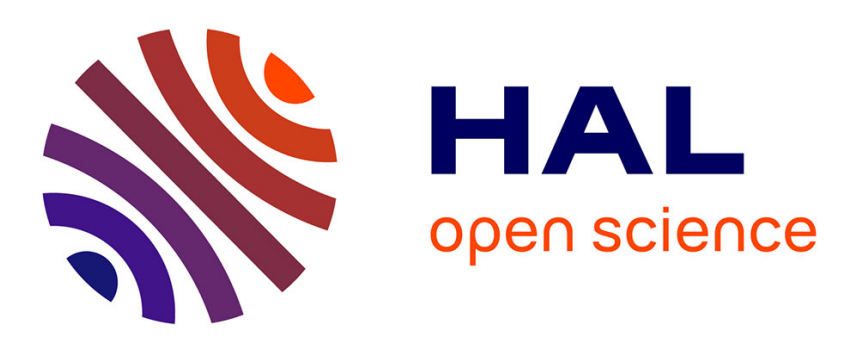

\title{
Electron thermalization and quantum decoherence in metal nanostructures
}

Rafal Jasiak, Giovanni Manfredi, Paul-Antoine Hervieux

\section{To cite this version:}

Rafal Jasiak, Giovanni Manfredi, Paul-Antoine Hervieux. Electron thermalization and quantum decoherence in metal nanostructures. Physical Review B: Condensed Matter and Materials Physics (1998-2015), 2010, 81 (24), pp.241401(R). 10.1103/PhysRevB.81.241401 . hal-00596640

\section{HAL Id: hal-00596640 \\ https://hal.science/hal-00596640}

Submitted on 30 May 2011

HAL is a multi-disciplinary open access archive for the deposit and dissemination of scientific research documents, whether they are published or not. The documents may come from teaching and research institutions in France or abroad, or from public or private research centers.
L'archive ouverte pluridisciplinaire HAL, est destinée au dépôt et à la diffusion de documents scientifiques de niveau recherche, publiés ou non, émanant des établissements d'enseignement et de recherche français ou étrangers, des laboratoires publics ou privés. 


\title{
Electron thermalization and quantum decoherence in metal nanostructures
}

\author{
R. Jasiak, G. Manfredi, and P.-A. Hervieux \\ Institut de Physique et Chimie des Matériaux de Strasbourg, 23 rue du Loess, BP 43, 67034 Strasbourg, France
}

(Received 15 March 2010; published 2 June 2010)

\begin{abstract}
The long-time dynamics of the electron gas in a thin metal film is studied using a microscopic phase-space model based on the quantum Wigner distribution. The model encompasses all relevant time scales from the femtosecond plasmon oscillations to the phonon-mediated coupling to the ionic lattice, which occurs on a picosecond time scale. The results are in good agreement with phenomenological estimates based on the two-temperature model, and correctly reproduce the main features observed in experiments on small sodium clusters. In particular, a classical relaxation time and a quantum decoherence time emerge naturally from the simulations.
\end{abstract}

DOI: 10.1103/PhysRevB.81.241401

PACS number(s): 73.50.-h, 03.65.Yz, 78.47.J-

\section{INTRODUCTION}

The electron dynamics in nanometer-sized metallic objects_-such as nanoparticles and thin films-has been widely investigated in the course of the last two decades. Indeed, thanks to the advent of ultrafast pump-probe spectroscopy, it has become routine to probe the electron response on a femtosecond time scale. Pump-probe experiments involve perturbing the system via a first stronger pulse, followed by a second weaker pulse that acts as a diagnostic tool. By modulating the delay between the pump and the probe, it is possible to assess with great precision the dynamical relaxation of the electron gas. ${ }^{1,2}$

In a typical experiment, the following schematic scenario is generally assumed. ${ }^{2}$ First, the electrons absorb quasiinstantaneously the laser energy via interband and/or intraband transitions; during this process the ionic background remains frozen and the electron distribution is nonthermal. Next $(\sim 100$ fs $)$, the injected energy is redistributed via electron-electron collisions, bringing the electron population to a metastable equilibrium at a temperature significantly higher than that of the lattice. On a picosecond time scale, the electron gas starts to interact incoherently with the phonons, and eventually relaxes to thermal equilibrium at the same temperature as the lattice. The lattice motion occurs on even longer time scales, ranging from tens of picoseconds (coherent "breathing" oscillations) to a few nanoseconds for the final heat transfer to the dielectric matrix in which the nano-objects are usually embedded.

As this evolution spans over an extremely wide time range, its comprehensive modeling-from laser excitation until matrix relaxation-is obviously a daunting task. Several past studies were devoted to the early stages of the electron dynamics, which is dominated by collective modes (plasmons) corresponding to coherent oscillations of the electron gas in the confining potential. Those studies relied on sophisticated microscopic models of the self-consistent electron dynamics, including Hartree-Fock calculations, ${ }^{3}$ time-dependent density-functional theory (TD-DFT), ${ }^{4}$ and phase-space approaches based on Wigner's pseudoprobability distribution. 5,6

For longer time scales, however, the situation is more problematic. On the one hand, the above models are compu- tationally very expensive, particularly for large systems. On the other hand, the long-time electron dynamics becomes dissipative, as the electrons exchange energy incoherently with the ion lattice via electron-phonon (e-ph) scattering. The modeling of dissipative effects is notoriously difficult in quantum mechanics, as the underlying theory is intrinsically Hamiltonian. Although some authors have recently attempted to include dissipative effects in TD-DFT calculations, ${ }^{7}$ there is as yet no broad consensus on how to construct a manybody quantum model that incorporates dissipation.

Alternatively, a number of phenomenological models ${ }^{8,9}$ — originally developed for bulk materials-have employed Boltzmann-type equations within the framework of the Fermi-liquid theory. Although such models incorporate e-ph collisions, they rely on the evolution of the global energy distribution, neglecting spatial profiles and the presence of interfaces. As such, they fail to describe the microscopic dynamics of finite-size systems.

Finally, at an even coarser level of description, the e-ph interaction can be modeled by a set of two coupled differential equations that describe the global evolution of the electron and lattice temperatures. ${ }^{10,11}$ Such two-temperature model (TTM) is widely used in the experimental literature on thin metal films, nanoparticles, ${ }^{2}$ and metal clusters ${ }^{12}$ and, despite its simplicity, is capable of reproducing with fair accuracy a large number of experimental results.

\section{MODEL}

In this Rapid Communication, we propose a model to describe the microscopic electron dynamics over all time scales up to the coupling with the ion lattice. In the same spirit of our previous works, ${ }^{5,6}$ the model is based on a phase-space description of the quantum electron dynamics, whereby the electron population is described by the Wigner pseudoprobability distribution $f\left(x, v_{x}, t\right) .{ }^{13}$ The advantage of this approach is that dissipative terms can be introduced by analogy with classical transport models, such as the FokkerPlanck (FP) equation.

We shall concentrate on a thin-film configuration and consider only excitations in the longitudinal direction (denoted $x$ ) normal to the film surfaces whereas the film size in the transverse directions is large compared to its thickness $L$. 
Under these conditions, a one-dimensional (1D) slab geometry can be assumed, ${ }^{7}$ although our model could be extended to two- and three-dimensional (3D) configurations with no conceptual difficulty, except for an increased computational effort. In the present work, we shall focus on alkali metals, particularly sodium, for which the influence of the core electrons can be neglected. In order to describe noble metals, a suitable model for $d$-band electrons should be implemented. This could be done, for instance, by replacing the fully occupied $d$ band by a continuous polarizable medium that screens the electron-electron and electron-ion Coulomb interactions inside the film. ${ }^{14}$

The Wigner distribution $f\left(x, v_{x}, t\right)$ evolves according to the following equation:

$$
\begin{aligned}
\frac{\partial f}{\partial t}+ & v_{x} \frac{\partial f}{\partial x}+\frac{i m_{e}}{2 \pi \hbar^{2}} \iint d \lambda d v_{x}^{\prime} e^{i m_{e}\left(v_{x}-v_{x}^{\prime}\right) \lambda / \hbar} f\left(x, v_{x}^{\prime}, t\right) \\
& \times\left[V_{\mathrm{eff}}\left(x+\frac{\lambda}{2}, t\right)-V_{\mathrm{eff}}\left(x-\frac{\lambda}{2}, t\right)\right]=\left(\frac{\partial f}{\partial t}\right)_{\mathrm{e}-\mathrm{ph}},
\end{aligned}
$$

where $m_{e}$ is the electron mass. $V_{\text {eff }}(x, t)$ is the effective potential, which is composed of a Hartree and an exchangecorrelation part. ${ }^{15}$ The Hartree potential obeys Poisson's equation $\partial^{2} V_{H} / \partial x^{2}=e^{2} / \varepsilon_{0}\left[\int f d v_{x}-n_{i}(x)\right]$, where $e$ is the absolute electron charge, $\varepsilon_{0}$ is the vacuum dielectric constant, and the ions form a motionless neutralizing background with density $n_{i}(x)$, which is equal to the bulk value $n_{0}$ inside the slab and goes rapidly to zero at the surfaces. ${ }^{5}$

The right-hand side of Eq. (1) constitutes our model for e-ph scattering and has the form of a classical FP term: $(\partial f / \partial t)_{\mathrm{e}-\mathrm{ph}}=D \nabla_{v}^{2} f+\gamma \nabla_{v} \cdot(\mathbf{v} G[f])$, where $\gamma$ is the nominal relaxation rate, $D$ is a diffusion coefficient in velocity space, and $G[\cdot]$ is a functional that depends on the statistics and on the dimensionality of the system. The FP equation can be seen as the continuum limit of the master equation of a Markovian process (random walk in velocity space).

For classical particles obeying an exclusion principle, the corresponding FP equation was derived by Kaniadakis et $a .^{16}$ For instance, $G[f]=f$ for particles obeying Maxwell-Boltzmann statistics and $G[f]=f(1-k f)$ for fermions in 3D. For fermions in $1 \mathrm{D}$, assuming that the electron distribution always remains a Fermi-Dirac one in the transverse directions $\left(v_{y}, v_{z}\right)$ and integrating the FP equation along these two directions, one obtains the expression $G[f]=f_{0}\left[1-\exp \left(-f / f_{0}\right)\right]$. Here $f_{0}=\frac{3}{4} \frac{n_{0}}{v_{F}} \frac{T_{i}}{T_{F}}, v_{F}$ and $T_{F}$ are the Fermi velocity and temperature, and $T_{i}$ is the lattice temperature. The preceding expression must be changed slightly to account for the fact that the Wigner distribution can be negative. Thus, to ensure that $G$ behaves identically for positive and negative values of $f$, one must have: $G[f]=f_{0}\left[1-\exp \left(-|f| / f_{0}\right)\right] \operatorname{sgn}(f)$.

It can be proven that $(\partial f / \partial t)_{\mathrm{e}-\mathrm{ph}}=0$ when the electron distribution is given by a 1D Fermi-Dirac function ( $\mu$ is the chemical potential),

$$
f_{e q}\left(v_{x}\right)=\frac{3}{4} \frac{n_{0}}{v_{F}} \frac{T_{i}}{T_{F}} \ln \left[1+\exp \left(-\frac{m_{e} v_{x}^{2} / 2-\mu}{k_{B} T_{i}}\right)\right],
$$

provided $D$ and $\gamma$ satisfy the relation $D / \gamma=k_{B} T_{i} / m_{e}$, where $k_{B}$ is the Boltzmann constant. Thus, the FP term ensures that the electron distribution relaxes to a classical Fermi-Dirac distribution with a temperature equal to that of the lattice. The ions are supposed to constitute a perfect reservoir with infinite heat capacity so that $T_{i}$ remains constant.

The electron relaxation rate can be written as $\gamma=g / c_{e},{ }^{12}$ where $g$ is the electron-phonon coupling constant appearing in the two-temperature model and $c_{e}$ is electron heat capacity. For an ideal Fermi gas (which is a good approximation for a metal film with thickness on the order of a few nanometers ${ }^{5}$ ), the heat capacity depends on the temperature as $c_{e}\left(T_{e}\right)=\pi^{2} n_{0} k_{B}\left(T_{e} / 2 T_{F}\right)$. In our case, the electron temperature is a time-dependent quantity that can be computed selfconsistently from the Wigner distribution $f$.

The only physical parameter that remains to be fixed is the electron-phonon coupling constant $g$. Recent experiments on nanometer-sized sodium clusters ${ }^{12}$ showed that $g$ is largely insensitive to the temperature and the size of the system. The measured value of the coupling constant was roughly $g=2 \times 10^{16} \mathrm{~W} / \mathrm{m}^{3} \mathrm{~K}$, which is about twice the experimental bulk value. All in all, we stress that our model does not contain any free fitting parameters and is entirely based on first-principles considerations and experimentally measured quantities.

\section{SIMULATIONS}

To study the electron dynamics, we first compute selfconsistently the ground state at finite initial temperature $T_{e}(0)=T_{i}$. Subsequently, the electron gas is perturbed by imparting a uniform velocity shift $\delta v$ on the initial Wigner distribution, which mimics the quasi-instantaneous dipolar interaction with the laser field. The ensuing dynamics is then followed for a certain duration.

The initial stages of the electron dynamics were studied in earlier works, ${ }^{5,6}$ and are consistent with the experimental scenarios described in Sec. I: the initial coherent oscillations of the electron gas are Landau damped and the excitation energy is converted into thermal energy, thus increasing the temperature of the electron gas up to several thousand degrees. After this phase (which lasts for approximately $100 \mathrm{fs}$ for sodium films of thickness $L=5-10 \mathrm{~nm}$ ), the electron gas is in a metaequilibrium state at a temperature much higher than that of the lattice. In this Rapid Communication, we are interested precisely in the energy exchanges between the electrons and the lattice.

In the numerical results, time is normalized to the inverse of the plasmon frequency $\omega_{p}=\left(e^{2} n_{0} / m_{e} \varepsilon_{0}\right)^{1 / 2}$, velocities to the Fermi speed $v_{F}$, distances to the Thomas-Fermi screening length $L_{F}=v_{F} / \omega_{p}$, and energies to the Fermi energy $E_{F}$. We concentrate on sodium films, for which $\omega_{p}^{-1}=0.11 \mathrm{fs}$, $L_{F}=0.12 \mathrm{~nm}$, and $T_{F}=3.62 \times 10^{4} \mathrm{~K}$. In the forthcoming simulations, the film thickness is $L=50 L_{F}$. The excitation ranges from $\delta v=0.06 v_{F}$ to $\delta v=0.35 v_{F}$ and corresponds to an excitation energy $E^{*} / E_{F}=\left(\delta v / v_{F}\right)^{2} L / L_{F}$. For $\delta v=0.1 v_{F}, E^{*}$ is approximately equal to the energy absorbed by a sodium nanoparticle of radius $R=1 \mathrm{~nm}$ irradiated by a laser pulse of intensity $I=10^{12} \mathrm{~W} \mathrm{~m}^{-2}$ for a duration $\tau=100 \mathrm{fs}$ $\left(E^{*}=I \tau \pi R^{2}\right) .{ }^{12}$ Although the numerical results reported in the next paragraphs were performed in the Hartree regime, we 


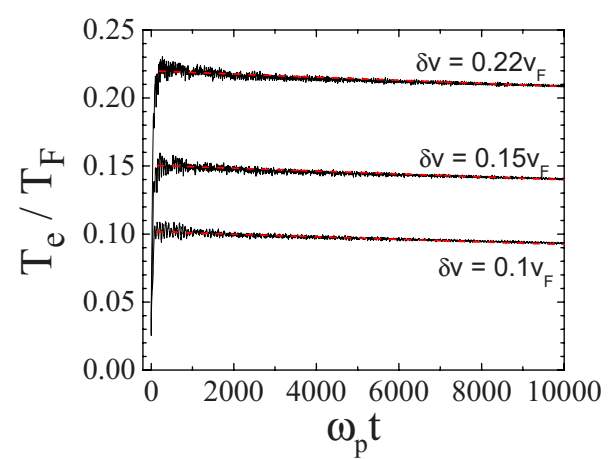

FIG. 1. (Color online) Time evolution of the electron temperature for three values of the excitation. The red dashed lines represent results obtained from the two-temperature model.

checked that the exchange-correlation potential does not alter any of the conclusions.

Figure 1 shows the evolution of the electron temperature for three values of the excitation. In all cases, the initial heating of the electron gas is clearly visible, with a peak temperature of a few thousand degrees. After the peak, the electrons start cooling down by exchanging energy with the phonon bath. The results of our Wigner calculations are compared with those obtained with a simple TTM, which describes the macroscopic evolution of the global electron temperature, $c_{e}\left(T_{e}\right) \dot{T}_{e}=-g\left(T_{e}-T_{i}\right)$, where the ion temperature is kept constant and the overdot stands for time differentiation. In order to model the laser excitation, the initial electron temperature is set to be equal to the peak temperature observed in the Wigner simulations. The agreement between the TTM and the Wigner simulations is quite impressive, and thus provides a direct confirmation of the validity of the TTM. This is an important issue, as the TTM is extremely popular as a phenomenological tool to interpret the electron relaxation curves obtained from experiments on thin metal films and nanoparticles. ${ }^{2}$

The temperature relaxation times $\tau_{R}$ observed in Fig. 2 are clearly on the order of a few picoseconds, although their actual values are difficult to estimate as the decay is so slow. Nevertheless, the trend can be extracted (Fig. 2) by using the running average of the electron temperature, defined as $\left\langle T_{e}\right\rangle(t)=\frac{1}{2 T} \int_{t-T}^{t+T} T_{e}\left(t^{\prime}\right) d t^{\prime}$ (with $\omega_{p} T=250$ ). This enables us to smooth out the fast oscillations and highlight the long-time trend. The relaxation time increases for larger perturbations,

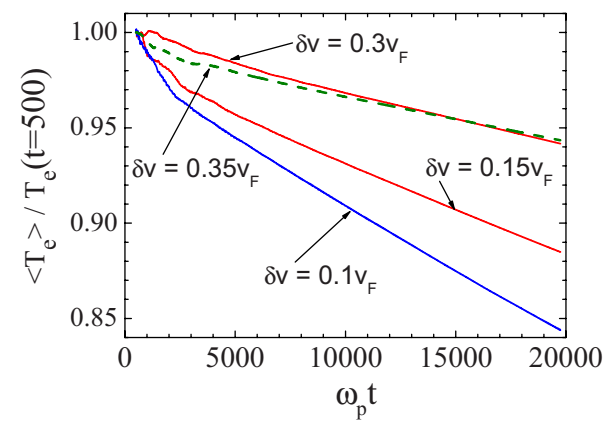

FIG. 2. (Color online) Time evolution of the running average of the electron temperature for various excitation energies.

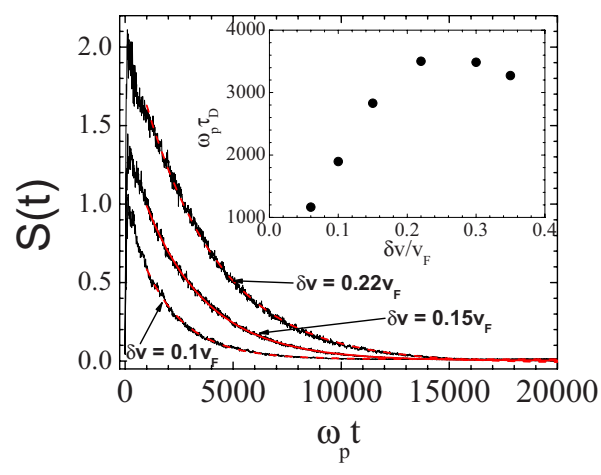

FIG. 3. (Color online) Evolution of $S(t)$ for different excitations. The red lines are exponential fits to the numerical curves. The inset shows the decoherence time as a function of the excitation.

as was observed in many experiments and simulations based on the TTM,,$^{11,12}$ and appears to saturate for large values of the excitation energy. ${ }^{17}$ This behavior may be linked to a quantum-classical transition. ${ }^{6}$ Indeed, for metal nanoparticles, the experimental relaxation time saturates with increasing particle's size,${ }^{18}$ which is another way to approach the classical regime.

The relaxation time measures the speed at which the energy is exchanged between the electrons and the lattice. Another relevant time scale is given by the decoherence time, which represents the typical time over which quantum correlations are lost to an external environment (here, the ion lattice). In a density-matrix language, the relaxation time corresponds to the decay of the diagonal terms whereas the decoherence time is related to the nondiagonal terms.

In order to evaluate the decoherence time, we recall that $f\left(x, v_{x}, t\right)$ can take negative values. The degree of "classicality" a Wigner distribution can be estimated from the weight of its negative parts, ${ }^{19}$ which leads us to define the quantity: $S(t)=\iint f_{<} d x d v_{x}$, where $f_{<}=-f$ when $f<0$, and zero elsewhere. The decoherence time $\tau_{D}$ was estimated by fitting $S(t)$ with a decaying exponential function (Fig. 3). Just like the relaxation time, the decoherence time also increases with increasing excitation energy, then saturates around $\delta v=0.2 v_{F}$. Interestingly, the excitation energy at this threshold is very close to the plasmon energy $\hbar \omega_{p} .{ }^{6}$

Finally, in order to highlight the decay toward a classical equilibrium, we show a cross cut of the electron Wigner distribution at the midpoint of the film, $f\left(x=0, v_{x}, \omega_{p} t=10^{4}\right)$, for two values of the initial excitation (Fig. 4). For $\delta v$ $=0.06 v_{F}$, the electron distribution is virtually identical to the Fermi-Dirac equilibrium $f_{e q}$. In particular, no appreciable negative values of $f$ are visible. In contrast, for $\delta v=0.3 v_{F}$ (corresponding to a longer decoherence time, see inset of Fig. 3) the Wigner distribution is still significantly far from equilibrium, and negative values are clearly present. ${ }^{19}$

\section{DISCUSSION}

From the above results, it appears that the electron-lattice interactions can be correctly described by two typical time scales, namely, the relaxation time $\tau_{R} \approx 3$ ps and the decoherence time $\tau_{D} \approx 0.25 \mathrm{ps}$ (these are often referred to as $T_{1}$ 


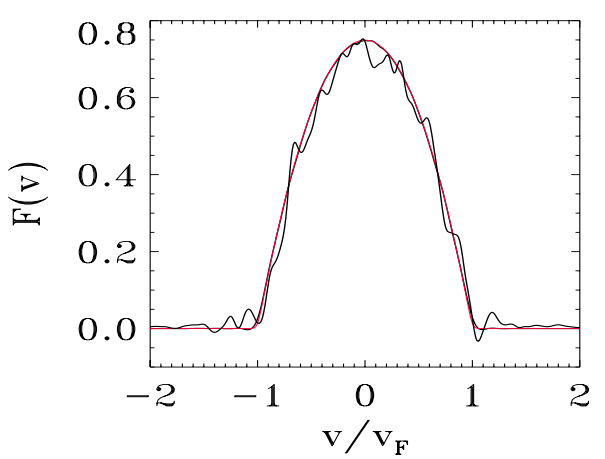

FIG. 4. (Color online) Wigner distribution at the midpoint of the film, for $\omega_{p} t=10^{4}$ and two values of the excitation. The red curve represents the Fermi-Dirac equilibrium $f_{\text {eq }}$. The distribution corresponding to $\delta v=0.3 v_{F}$ displays oscillations whereas the $\delta v=0.06 v_{F}$ case is indistinguishable from $f_{e q}$.

and $T_{2}$ in the semiconductor literature $\left.{ }^{20}\right)$. The relaxation time observed in the simulations is in good agreement with that measured in experiments on small sodium clusters. ${ }^{12}$

The overall electron thermalization thus occurs in two stages. First, during the decoherence process, the Wigner distribution loses its negative values (thus becoming a bona fide probability density) and tends to a classical Fermi-Dirac function with temperature $T_{e} \gg T_{i}$. This is consistent with experimental observations, which show a thermal distribution of emitted electrons (with $T_{e}$ a few thousand degrees) soon after the laser irradiation of sodium clusters. ${ }^{21}$ Subsequently, the electron gas cools down until it reaches the lattice temperature. This relaxation process occurs on a time scale $\tau_{R} \gg \tau_{D}$

In summary, we have proposed a microscopic model to include the e-ph coupling in the self-consistent electron dynamics of nanometer-sized metallic objects. The model reproduces semiquantitatively recent experimental results on sodium clusters and is in good agreement with popular phenomenological approaches such as the two-temperature model. The decoherence and relaxation times emerge naturally from the equations and their scalings with the excitation energy is in accord with experimental findings.

\section{ACKNOWLEDGMENTS}

This work was performed using computing resources from IDRIS (Grant No. i2009051819). We also acknowledge funding from the Agence Nationale de la Recherche, Project No. ANR-06-BLAN-0059.
${ }^{1}$ C.-K. Sun, F. Vallée, L. H. Acioli, E. P. Ippen, and J. G. Fujimoto, Phys. Rev. B 50, 15337 (1994).

${ }^{2}$ J.-Y. Bigot, V. Halté, J.-C. Merle, and A. Daunois, Chem. Phys. 251, 181 (2000).

${ }^{3}$ U. Schwengelbeck, L. Plaja, L. Roso, and E. Conejero Jarque, J. Phys. B 33, 1653 (2000).

${ }^{4}$ F. Calvayrac, P.-G. Reinhard, E. Suraud, and C. Ullrich, Phys. Rep. 337, 493 (2000).

${ }^{5}$ G. Manfredi and P.-A. Hervieux, Phys. Rev. B 72, 155421 (2005); Opt. Lett. 30, 3090 (2005).

${ }^{6}$ R. Jasiak, G. Manfredi, and P.-A. Hervieux, New J. Phys. 11, 063042 (2009).

${ }^{7}$ R. D'Agosta and G. Vignale, Phys. Rev. Lett. 96, 016405 (2006).

${ }^{8}$ B. Rethfeld, A. Kaiser, M. Vicanek, and G. Simon, Phys. Rev. B 65, 214303 (2002).

${ }^{9}$ C. Voisin, N. Del Fatti, D. Christofilos, and F. Vallée, J. Phys. Chem. B 105, 2264 (2001).

${ }^{10}$ H. E. Elsayed-Ali, T. B. Norris, M. A. Pessot, and G. A. Mourou, Phys. Rev. Lett. 58, 1212 (1987).

${ }^{11}$ G. V. Hartland, Phys. Chem. Chem. Phys. 6, 5263 (2004).
${ }^{12}$ M. Maier, G. Wrigge, M. A. Hoffmann, P. Didier, and B. v. Issendorff, Phys. Rev. Lett. 96, 117405 (2006).

${ }^{13}$ M. Hillery, R. F. O'Connell, M. O. Scully, and E. P. Wigner, Phys. Rep. 106, 121 (1984).

${ }^{14}$ B. Palpant, B. Prével, J. Lermé, E. Cottancin, M. Pellarin, M. Treilleux, A. Perez, J. L. Vialle, and M. Broyer, Phys. Rev. B 57, 1963 (1998).

${ }^{15}$ L. Brey, J. Dempsey, N. F. Johnson, and B. I. Halperin, Phys. Rev. B 42, 1240 (1990).

${ }^{16}$ G. Kaniadakis and P. Quarati, Phys. Rev. E 48, 4263 (1993).

${ }^{17}$ V. Halté, J. Guille, J.-C. Merle, I. Perakis, and J.-Y. Bigot, Phys. Rev. B 60, 11738 (1999).

${ }^{18}$ J. Lermé, G. Celep, M. Broyer, E. Cottancin, M. Pellarin, A. Arbouet, D. Christofilos, C. Guillon, P. Langot, N. Del Fatti, and F. Vallée, Eur. Phys. J. D 34, 199 (2005).

${ }^{19}$ S. Deléglise, I. Dotsenko, C. Sayrin, J. Bernu, M. Brune, J.-M. Raimond, and S. Haroche, Nature (London) 455, 510 (2008).

${ }^{20}$ H. O. Wijewardane and C. A. Ullrich, Appl. Phys. Lett. 84, 3984 (2004).

${ }^{21}$ R. Schlipper, R. Kusche, B. v. Issendorff, and H. Haberland, Appl. Phys. A 72, 255 (2001). 